\title{
Host contribution to parasite persistence is consistent between parasites and over time, but varies spatially
}

\author{
Jon Bielby, ${ }^{1,6}$ Stephen J. Price iD ${ }^{2,3}$ Camino Monsalve-Carcaño, ${ }^{4}$ and Jaime Bosch id 4,5 \\ ${ }^{1}$ School of Natural Sciences and Psychology, Liverpool John Moores University, James Parsons Building, Byrom Street, Liverpool L3 \\ 3 AF United Kingdom \\ ${ }^{2}$ UCL Genetics Institute, Darwin Building, Gower Street, London WC1E 6BT United Kingdom \\ ${ }^{3}$ Institute of Zoology, Zoological Society of London, Regents Park, London NW1 4RY United Kingdom \\ ${ }^{4}$ Museo Nacional de Ciencias Naturales, CSIC, José Gutiérrez Abascal 2, Madrid 28006 Spain \\ ${ }^{5}$ Research Unit of Biodiversity (CSIC, UO, PA), Oviedo University-Campus Mieres, Mieres, Spain
}

Citation: Bielby, J., S. J. Price, C. Monsalve-Carcaño, and J. Bosch. 2020. Host contribution to parasite persistence is consistent between parasites and over time, but varies spatially. Ecological Applications. 00(00): e02256. 10.1002/eap.2256

Abstract. Most parasites and pathogens infect multiple hosts, but a great deal of variation exists in the role of those hosts in persistence of infection. Understanding which hosts are most important in maintaining parasites can provide a clearer target for infection control. Recently developed empirical and theoretical approaches provide a way to quantify the relative contribution of hosts within a community and place them in a multi-host framework to better direct control efforts. Amphibians provide a framework for better understanding multi-host-multiparasite dynamics. Two well-studied amphibian parasites, Batrachochytrium dendrobatidis (Bd) and Ranavirus, infect multiple host species and exhibit a great deal of heterogeneity in how they affect hosts. We used these two parasites and a community of five amphibian species to investigate the relative importance of hosts in parasite persistence, and how any patterns varied spatially and temporally. At two sites (Lake Ercina and Lake Lloroza in the Picos de Europa National Park, Spain) we collected data on the prevalence and shedding rate of parasite infection for both Bd and Ranavirus, and the abundance of each species' life stages. We used these data to parameterize a recently developed modeling framework, which was used to quantify the relative contribution of each host to the community reproductive number, $R_{0}$. By comparing each host-category over time and between sites we were able to identify consistencies in which host was responsible for the maintenance of these two parasites. Within a site one species consistently contributed the most to the persistence of both parasites. This consistency did not transfer between sites, the maintenance host species being different for each. At one site (Ercina), life stages of the common midwife toad, Alytes obstetricans, acted as the maintenance host for both Bd and Ranavirus. In contrast, at the second site, Lloroza, the alpine newt, Ichthyosaura alpestris, fulfilled that role. A single host species was responsible for infection persistence of both parasites at each lake. Attempts to control the infection levels and impacts of multiple parasites can benefit from a community epidemiology approach, and provide clarity on which hosts are the foci of mitigation efforts. However, at a small spatial scale, the target host may vary according to the physical qualities of those sites and the demographics of the host community.

Key words: amphibian; Batrachochytrium dendrobatidis (Bd); epidemiology; multi-host; pathogen; Picos de Europa National Park; ranavirus; $\mathrm{R}_{0}$.

\section{INTRODUCTION}

Understanding of multi-host, multi-pathogen systems has greatly increased in recent years, but there remains a lack of information on how parasites are maintained within a community of hosts and, perhaps most importantly, how to identify which species drive transmission within a community (Rudge et al. 2013). Recent

Manuscript received 30 January 2020; revised 11 June 2020; accepted 16 August 2020. Corresponding Editor: Trenton W. J. Garner.

${ }^{6}$ E-mail: j.n.bielby@ljmu.ac.uk conceptual (Fenton and Pedersen 2005) and mathematical (Fenton et al. 2015) frameworks have been developed to better identify where hosts within a community stand in terms of their role in parasite persistence. Using these frameworks, we can identify where communities lay on a multi-host spectrum. At one extreme a community may rely heavily on spillover from one or more of its constituent species, at the other a community of species may be truly multi-host with multiple species or life stages contributing to parasite persistence (Holt et al. 2003, Fenton and Pedersen 2005, Fenton et al. 2015). The ability to place communities and their members within this 
broad framework would help us to devise more directed intervention strategies in complex situations (Lembo et al. 2008).

A key measure in quantifying an individual's or species' role in the spread and persistence of infection is that of the basic reproductive number $\left(R_{0}\right)$ of a parasite (Anderson and May 1992). This parameter describes the ability of a parasite to invade a population (or community) of naive hosts, and provides a threshold for understanding the circumstances in which infection will spread: if $R_{0}$ of an infection is below 1 , the infection will tend to fade out, whereas if $R_{0}$ is above 1 , it will have a high likelihood of persisting and potentially spreading. Therefore, measuring $R_{0}$ of a parasite provides us with both a metric for quantifying parasite spread, and a target at which we can aim attempts to reduce infection transmission and promote fade-out.

The $R_{0}$ of a parasite may vary in response to a number of variables related to the ecological context in which it finds itself. Considerable variation in host-parasite dynamics may be introduced from many different sources including, but not limited to, host community structure (Fernández-Beaskoetxea et al. 2016), host density (Rachowicz and Briggs 2007), microfaunal community of both the environment (Schmeller et al. 2014) and the host (Bates et al. 2018), seasonality (Clare et al. 2016), and temperature (Price et al. 2019), both independently and via interactions and correlative relationships between them. For a given parasite, understanding how these sources of variation affect $R_{0}$ within a community and whether robust rules of thumb exist, would enable us to identify when knowledge of a system is transferable and when extra information is required to inform our understanding of parasite persistence. For example, understanding whether and when the $R_{0}$ of community members varies seasonally, and/or between sites could inform targeted management options. Understanding the contribution species play in infection dynamics across different sites and over time may be further complicated if we consider the dynamics of multiple parasites within a community of hosts.

In addition to most parasites being able to infect multiple host species, most hosts harbor multiple parasites (Knowles et al. 2013). In any community of hosts, parasites may interact within the host in different ways depending upon the niche they fill (Pedersen and Fenton 2007): parasites may interact directly via mechanisms such as competition or resource release (Griffiths et al. 2014), or indirectly via the host immune system bestowing cross-immunity or facing energetic trade-offs on different arms of the immune system (Fenton 2008, Jolles et al. 2008, Ezenwa et al. 2010). These direct and indirect interactions can therefore promote the persistence of a community of parasite species, or may benefit the $R_{0}$ of one parasite to the detriment of another. We currently have a poor understanding of how parasite-interactions translate into broader patterns of host roles within a community. Understanding where species are placed within a multi-host framework, and how this varies for different pathogens in the same system will be a useful first step in understanding the dynamics of a community of hosts and parasites, which likely represents the default setting in most ecological systems.

Batrachochytrium dendrobatidis (Bd) is a generalist parasite of amphibians that has been strongly associated with species declines and extinctions over recent decades (O'Hanlon et al. 2018). The flexibility of this parasite in both host range and environmental requirement is illustrated by its near global distribution, and the fact that it has infected amphibians from all three amphibian Orders in 56 of the 82 countries in which hosts have been sampled (Martel et al. 2018). Within this host range there is considerable inter- and intraspecific variation in key epidemiological parameters and in the effects of parasite exposure. While certain species seem predisposed to becoming infected others seem much more resistant to carrying detectable infections (Balaž et al. 2014). Similarly, different populations of the same species may exhibit different responses to infection; some populations allow long-term pathogen persistence, others may experience pathogen fade-out or host decline and even extinction (Tobler and Schmidt 2010, Tobler et al. 2012). While several routes of dispersal and transmission have been identified in Bd (e.g., Garmyn et al. 2012, Kolby et al. 2015, Martel et al. 2018) the infectious life stage of the parasite is a waterborne zoospore, which suggests that environmental transmission is an important route of transmission between members of aquatic amphibian communities.

Ranaviruses (Rv) can also be a cause of severe amphibian declines (Teacher et al. 2010, Price et al. 2014, Rosa et al. 2017), and have a very broad host range that includes two other vertebrate classes (fish and reptiles; Price et al. 2017) although different strains vary in the degree of generalism they exhibit. Viruses in the FV3 lineage are capable of infecting common frogs (Rana temporaria) and common toads (Bufo bufo) in the UK but appear more virulent to the former (Duffus et al. 2015, Price et al. 2017)). Inter-host dynamics may be an important factor in the epidemiology of infection; the presence of $B$. bufo is associated with an increased likelihood but reduced severity of disease outbreaks in $R$. temporaria (North et al. 2015). By contrast, common midwife toad ranaviruses (CMTV) in the Picos de Europa National Park, Spain routinely exhibit lethal effects across a very broad range of amphibian hosts and have been implicated in mass-mortality incidents involving the entire amphibian community there (both anurans and caudates; Price et al. 2014), but little is known about the route of viral transmission and nothing is known about the relative contributions of each host species to the maintenance and transmission of infection. The broad host range of $\mathrm{Rv}$ in a community is consistent with infection transmission via an environmental pool of particles, as direct contact rates between species and life stages are unlikely to be high. 
In $\mathrm{Bd}$ and $\mathrm{Rv}$, amphibians are therefore the hosts for two high-profile, generalist parasites that exhibit a large degree of inter- and intraspecific variation in host response. Further, host response may also vary among life stages within a species. Quantifying the degree to which species or life stages within a community contribute to infection persistence both over time and at different sites would allow us to better understand where differences and consistencies lay in community infection dynamics. If data suggest consistencies in which hosts contribute the most to the maintenance of infection (hereafter "maintenance hosts"), those species or life stages could be used as foci for intervention and mitigation.

Here, we use empirical field data and a recently developed mathematical model (Fenton et al. 2015) to quantify how members of a community of hosts contribute to the persistence of infection for both $\mathrm{Bd}$ and $\mathrm{Rv}$. We compare the results obtained across the months of a year and between two field-sites that differ ecologically, thereby including spatial and temporal components to our analyses. We pay particular attention to those months at which host populations, and therefore hostpathogen dynamics, are most likely to meet assumptions of equilibrium of infection levels. Using these data, we aim to place species within a framework describing the role of different hosts within a community and draw out any commonalities and differences that exist. If there are strong trends in the placement of species or life stages within this framework across seasons, between sites and between the two focal parasites, it may hint that controlling infection can be more temporally geographically and ecologically transferable. Alternatively, differences in the role hosts play in parasite persistence suggest that intervention measures will be heavily context dependent as a result of aspects of the site and its host community.

\section{Methods}

\section{Study system}

The Picos de Europa National Park (PNPE; hereafter the "study system") is a protected wilderness area comprised of limestone mountains in the north of Spain. It is an important wildlife area but is also used for recreational activities and stockbreeding. There are five species of amphibian that are commonly found in the park that spend at least some of their annual activity cycle in the aquatic environment (common midwife toad, Alytes obstetricans [Ao]; spiny toad, Bufo spinosus [Bs]; alpine newt, Ichthyosaura alpestris [Ia]; palmate newt, Lissotriton helveticus [Lh]; and the common frog, Rana temporaria $[\mathrm{Rt}])$. In addition, there are a small number of rarer species that occur much less frequently, at a much lower abundance. Outbreaks of ranavirosis were first observed in the park in 2005 and resulted in severe and dramatic declines of some hosts at several sites (Price et al., 2014). $\mathrm{Bd}$ has also been present in the region since at least 2005 without observations of chytridiomycosis (Price et al. 2014). Both Bd and $\mathrm{Rv}$ can infect all of these species, and can infect their constituent life stages (which for the purposes of these analyses we categorize as larva, metamorphic individual, and, as a single category, juvenile and adults). Hereafter, for simplicity, the combinations of host species and life stages are referred to as "host categories" (i.e., a species will have as many host categories as it has life stages sampled in the study).

In this study, two lakes (Ercina and Lloroza, in the Picos de Europa National Park, Spain, hereafter the "study sites" to distinguish them from our study system) were sampled to allow us to compare infection dynamics in locations with different biotic and abiotic qualities. Ercina is a lake at $1,110 \mathrm{~m}$ above sea level with a maximum surface area of approximately 6.6 ha and a maximum depth of approximately $3 \mathrm{~m}$. Lloroza is a pond, or small lake at 1,860 $\mathrm{m}$ above sea level, with a surface area of approximately $3070 \mathrm{~m}^{2}$ in summer that can dry out completely over summer in the absence of rain. All sampling for this study took place between March and September 2017, and during this period Lloroza was approximately $1,300 \mathrm{~m}^{2}$ in area.

\section{Amphibian surveys}

Both lakes were surveyed throughout the whole reproductive period of amphibians at the sites from egg deposition through to metamorphosis. The period of sampling was shorter at Lloroza than Ercina, as the higher elevation of the former leads to a shorter period of amphibian activity there. At Ercina, we collected data for the months March-September, whereas at Lloroza, due to the shorter period of amphibian activity, data were collected for May-September.

At both sites, lakeside walking transects were conducted to estimate the abundance of each species. Each transect was completed by a pair of observers at twilight when amphibian activity is highest. At each lake, the lakesides were split into four sections. Observers travelled at a consistent walking speed to maintain a standardized sampling effort and counted every animal in the water up to a distance of $2 \mathrm{~m}$ from the shore, and every animal out of the water up to a distance of $1 \mathrm{~m}$ from the shore. At Lloroza, the entire lake shore was surveyed, whereas at Ercina, only half of the lake could be surveyed as the remainder was inaccessible due to vegetation growth. Both observers independently counted every animal seen at each section resulting in a total estimate of abundance for each host-category at each section of lakeside on the date of the survey. For the two estimates obtained, if the lower estimate was $<50 \%$ of the higher estimate, the observers would repeat the transect for that section of lakeside. If the lower estimate was within $50 \%$ of the higher estimate the mean value was calculated and used as a measure of abundance. Using this protocol maximized the likelihood of collecting accurate enough data to yield a robust 
estimate of species abundance and obtain a suitable number of samples for diagnostic parasite screening.

\section{Sampling for detection of parasites}

All observed life stages of host amphibian species were sampled at each site in each month to detect the presence of parasite infection and infection load. For the purposes of this study, we assumed that $\mathrm{Rv}$ infection was via environmental or amphibian hosts and did not include alternative taxa (e.g., fish or reptiles) in our data collection or analyses. For each life stage and species, as many individuals as possible were sampled during a particular survey, exhaustively for those sampled via swabs, and up to a maximum of 20 in the case of tissue samples. As tissue samples are preferred for diagnosis of RV tail clips were taken from most life stages (larvae of both anurans and urodeles, and metamorphic individuals, juveniles and adult urodeles), while toe-clips were taken from juvenile to adult anurans. Tissue samples were used for diagnosis of both $\mathrm{Rv}$ and $\mathrm{Bd}$, except for $\mathrm{Bd}$ in anuran larvae, which only become infected in their oral disc. For midwife toad (A. obstetricans) larvae an oral disc swab was taken for $\mathrm{Bd}$ diagnosis, and a tail clip taken for $\mathrm{Rv}$ detection. Since spiny toads (B. spinosus) and common frogs ( $R$. temporaria) have smaller larvae, oral swabs are not a reliable method of sampling, and tail clips would compromise animal welfare. Individuals were therefore humanely euthanized using an overdose of MS222 (5 g/ $\mathrm{L}$ ), and the whole oral disc was used for detection of $\mathrm{Bd}$. All tissue samples were fixed in $70 \%$ ethanol. Swabs were kept dry and refrigerated.

DNA was extracted from tissue samples using DNeasy Blood and Tissue Kit (Qiagen, Hilden, Germany) following the manufacturer's protocol. DNA was obtained from swabs with PrepMan Ultra (Thermo Fisher Scientific Inc, Waltham, MA, USA) following Hyatt et al. (2007).

The qPCR for $\mathrm{Bd}$ and $\mathrm{Rv}$ was performed in the MNCN-CSIC lab following Boyle et al. (2004; Bd) and Leung et al. (2017; RV) respectively on a MyGo Mini PCR machine (IT-IS Life Science Ltd, Dublin, Republic of Ireland). Negative controls and standards with known concentrations of $\mathrm{Bd} / \mathrm{Rv}$ were used in each plate.

These tests yielded a measure of whether an individual was infected or not, and the infection intensity of each infected individual. The former was used to calculate the prevalence of infection for that host-category, and the latter was taken as a surrogate measure of infection shedding per infected host. For some host categories the prevalence of infection was calculated from a very small sample size, which could be inaccurate and heavily skew the estimates of $R_{0}$ obtained. The high influence of prevalence of infection in our data largely resulted in very high estimates of $R_{0}$ for those host categories. To account for this, we calculated confidence intervals for each prevalence estimate; the Clopper-Pearson exact confidence intervals for the prevalence of infection of a host-category were calculated using the $\mathrm{R}$ function exactci() in the PropCIs library (R Core Team 2019). In addition to calculating the $R_{0}$ of each host-category using the central estimate of infection prevalence of that host-category, we also made the calculation of $R_{0}$ contribution using the lowest estimated prevalence within those $95 \%$ confidence intervals (i.e., the lower $5 \%$ confidence interval estimate). Hereafter we refer to the former as the "central $R_{0}$ estimate" and the latter as the "conservative $R_{0}$ estimate" for each host-category, but we focus our reporting and interpretation on the latter as the results are qualitatively the same (Supplementary information).

Data on infection intensity per individual was logtransformed prior to analysis because of the heavily skewed nature of parasite infection data, with many low values and a small number of very heavy infections. This data treatment also helped avoid estimated $R_{0}$ contributions being heavily affected by inaccurate estimation of absolute infection load at the high-end of the scale that lie well outside the range of the Bd qPCR controls.

The data used to calculate the contribution toward community $R_{0}$ at Ercina and Lloroza for $\mathrm{Bd}$ and $\mathrm{Rv}$ are available as Supporting Information (Metadata S1 and Data S1). These data are host-category abundance, infection prevalence, and infection shedding rate, as described in the following subsections.

\section{Quantifying species' contribution to parasite persistence}

We used the prevalence-based approach of Fenton et al. (2015) to quantify the contribution of each hostcategory to the persistence of Bd and Rv. Briefly, Fenton et al. generalized the work of Funk et al. (2013) and Rudge et al. (2013) so that quantifying the contribution of each host-category to the $R_{0}$ of a parasite relied only on parameters that are routinely collected in field studies. The methods developed in these papers are derived from a (S)usceptible-(I)nfected-(E)nvironmental pathogen transmission model (Barbour 1996), which makes a number of key assumptions relevant to these analyses, which we outline here. First, the method assumes that the process of loss and gain of infection, and therefore the observed prevalence of infection are at a steady state, that is to say that the dynamics of the system are at equilibrium. Given that both Bd and RV have been present at this particular site for over a decade (since 2005), infection with both parasites appears to be endemic. However, because of the highly seasonal nature of infection dynamics at our study site, at different times of the year the prevalence of infection within a given host-category may vary (i.e., may not be at equilibrium). For this reason we have focused our analysis and discussion mainly on the two time points of the year at which amphibian densities and infection levels are most likely to be stable; these time points would be at the beginning of the amphibian activity cycle, before an influx of susceptible individuals enters the population via 
reproduction, and at the end of the year when any individuals samples have had a full cycle of activity to become infected.

Another key assumption of the model framework is that infection occurs via a single pool of infectious stages in the environment. For Bd waterborne infectious, zoospores are an important source of infection. Further, within the focal ponds the broad host range of both parasites suggests that an environmental pool of infection is likely to be a large contributor to the transmission of infection (although it is unlikely to be the only route of infection). However, there still remains the possibility that direct transmission is an important route of infection transmission for $\mathrm{Bd}$, and recent research on $\mathrm{Rv}$ suggests that environmental transmission may contribute a relatively small proportion toward infection transmission (Brunner and Yarber 2018). To relax the assumption that transmission occurred solely by environmental transmission we therefore also modeled transmission directly using the amended "direct transmission" formula as outlined by Fenton et al. (2015; Eq. S.1 in Appendix S1). Both of these assume that transmission of infection increases in a density-dependent fashion with transmission rates increasing as a linear function of density.

For a given community, calculating the $R_{0}$ contribution of member host categories requires information on the prevalence of parasite infection $(P)$ and the abundance of each host-category $(H)$, as well as the release rate of parasite infective stages per infected individual $(\lambda)$, and the rate of parasite infection transmission ( $\beta$ ). Values for parameters $P, H$, and $\lambda$ were estimated from the amphibian data described in Sampling for detection of parasites.

These variables combine to provide the composite parameters introduced in Table 1 and below, which themselves are used to give Eq. 1, used to calculate the contribution to the $R_{0}$ of a parasite for a focal host-category $i$ (where $n$ is the total number of host categories sampled within that focal site-season combination)

$$
R_{0, i}=\frac{1}{\left(1-P_{i}\right) \sum_{j=1}^{n}\left(\delta^{i j} \varepsilon^{i j} \nu^{i j} \omega^{i j}\right)} .
$$

In brief, $\delta_{\mathrm{ij}}$ represents the ratio of parasite infective stages produced in host-category $j$ relative to those from focal host-category $i\left(\delta_{\mathrm{ij}}=\lambda_{\mathrm{j}} / \lambda_{\mathrm{i}}\right), \epsilon$ represents the ratio of species abundances $\left(\varepsilon_{\mathrm{ij}}=H_{j} / H_{i}\right)$, and $\mathrm{v}_{\mathrm{ij}}$ is the ratio of infection prevalence in host-category $j$ relative to hostcategory $i\left(\mathrm{v}_{\mathrm{ij}}=P_{j} / P_{i}\right)$.

Because of difficulties in directly measuring the rate of parasite transmission, $\beta$, we ran a sensitivity analysis to explore a range of feasible, if extreme, values of the composite parameter $\omega_{i j}$, which measures the ratio of inter- vs. intra-host-category transmission $\left(\beta_{i j} / \beta_{i i}\right)$. We explored parameter space running from a
Table 1. Description of variables used in Eq. 1.

\begin{tabular}{|c|c|}
\hline Variable & Description \\
\hline$P_{i}$ & prevalence of infection for host-category $i$ \\
\hline$H_{i}$ & abundance of host-category $i$ \\
\hline$\lambda_{i}$ & $\begin{array}{l}\text { release of parasite infective stages produced per } \\
\text { infected individual within a host-category } i\end{array}$ \\
\hline$\beta_{i j}$ & $\begin{array}{l}\text { rate of parasite transmission for host-category } i \text { (i.e., } \\
\text { the rate at which } i \text { picks up infectious particles } \\
\text { released by host-category } j \text { ) }\end{array}$ \\
\hline$\delta_{i j}$ & $\begin{array}{l}\text { ratio of release of parasite infective stages produced } \\
\text { in host-category } j \text { relative to those from the focal } \\
\text { host-category } i\left(\delta_{i j}=\lambda_{j} / \lambda_{i}\right)\end{array}$ \\
\hline$\varepsilon_{i j}$ & $\begin{array}{l}\text { ratio of abundance of host-category } j \text { relative to } \\
\text { host-category } i\left(\varepsilon_{i j}=H_{j} / H_{i}\right)\end{array}$ \\
\hline $\mathrm{v}_{i j}$ & $\begin{array}{l}\text { ratio of infection prevalence in host-category } j \\
\text { relative to host-category } i\left(v_{i j}=P_{j} / P_{i}\right)\end{array}$ \\
\hline$\omega_{i j}$ & $\begin{array}{l}\text { ratio of between host-category transmission rate } \\
\text { relative to within host-category transmission rate } \\
\left(\omega_{i j}=\beta_{i j} / \beta_{i i}\right)\end{array}$ \\
\hline
\end{tabular}

situation in which there was negligible transmission from other host categories to the focal host $\left(\omega_{i j} \sim 0\right)$ to a scenario in which inter and intra-host-category transmission were equal $\left(\omega_{i j}=1\right)$. By doing so, following Fenton et al., we effectively ask the question: what level of $R_{0, i}$ is required to generate the empirical prevalence in that focal host-category under different degrees of interto intra-host-category transmission? Because the denominator contains the term $1-P_{i}$, we set an upper bound of 0.99 to the parameter of $P_{i}$ to avoid dividing by the number zero.

\section{Model outputs and interpretation}

Comparison of $\mathbf{R}_{0}$ contribution across time and space for two parasites.-Using the results obtained, we plotted for each site and each month of the year the $R_{0}$ contribution of each host-category of host for both Bd and Rv on the same plot. These plots allowed comparison of how much host categories contributed to the maintenance of both parasites when time and site were held constant. Trends over the months of the year and between sites could then be assessed by visual comparison of the different resulting plots.

To analyze whether host-category contribution to $R_{0}$ of the parasites was consistent at the species level, the data on separate life stages were aggregated and we tallied which of five species contributed most to the $R_{0}$ for each site-month combination (hereafter we refer to the most highly contributing species as the "maintenance host"). Binomial tests were used to determine whether the number of times a species had the highest estimated $R_{0}$ was randomly distributed across the five species (i.e., an exact null probability of $1 / 5=0.2$ ) or, alternatively, whether there was a taxonomic pattern in which species contributed the most to the $R_{0}$ of parasites within their community. 


\section{Results}

\section{Patterns of $\mathrm{R}_{0}$ in values early and late in the amphibian activity cycle}

The species contributing most to the persistence of infection within the community was consistent within a site for the first and last month of the sampling season. However, the species differed between sites (at Ercina, the most heavily contributing species was Ao; at Lloroza, it was Ia). In all cases, the lower limit of the $R_{0}$ estimate confidence intervals were greater than 1.0 , suggesting the identity of the hosts with the highest $R_{0}$ estimates (i.e., maintenance hosts) are robust to changes in inter-species transmission rates. Combined, these results suggest that when the system is most likely to be at equilibrium levels of infection, a single species is the key host in maintaining infection within the two communities, but that the key species within each community differs. These results reflect the overall pattern when data collected in other months is included and analysed, and in this section we will present the results obtained from analyses including all months.

\section{Broader patterns in the $\mathrm{R}_{0}$ values of maintenance hosts}

There were 23 site-month-parasite combinations (14 cells in Table 2, 9 cells in Table 3, with one cell omitted because of a lack of infected individuals being detected). For 22 of 23 site-month-parasite combinations, a single host-category contributed an $R_{0}$ that was in excess of the key threshold of 1 regardless of the relative level of intervs. intra-host-category transmission (see Fig. 1 and 2). In the remaining case the $R_{0}$ estimates of the maintenance host-category (which we define as the host-category with the largest contribution to $R_{0}$ ) straddled the threshold value of 1.0 across the transmission parameter values used; in the month of June, Ia adults were the maintenance host-category in contributing to the persistence of $\mathrm{Bd}$ in Lloroza with a mean $R_{0}=0.97$ with values obtained ranging from 0.93 to 1.02 . Tables 2 and 3 highlight the mean value of $R_{0}$, and the range of values obtained across all values of our transmission sensitivity analysis.

At the site level, the same host-category consistently had the highest $R_{0}$ estimates for both parasites at a given time-point. At Ercina, for five of the seven months in which we collected data, the maintenance host-category was the same for both parasites (Table 2). At Lloroza, the same was true for two of the five months in which data were collected (Table 3). At Ercina, the host species was Ao, whereas at Lloroza the host species was Ia.

Table 2 shows that, at Ercina, Ao adults were the most highly contributing host in five month-parasite combinations, Ao larvae in five, Ao metamorphs in two, Bs in one, and Ia in one. When split by parasite at this site, Ao larvae were the largest contributors to $\mathrm{Bd}$ persistence in two months, Ao metamorphs in one, Ao adults in three, and Bs adults in one. For Rv, Ao larvae contributed the largest amount toward community $R_{0}$ in three months, Ao metamorphs in one, Ao adults in one month, and Ia in one month. At Lloroza the data suggest that Ia was the host contributing the most to community $R_{0}$ in eight of the nine month-parasite combinations in which animals were sampled (Table 3 ). Ia adults were maintenance in five of those nine, Ia larvae in three, Bs metamorph in one month, and in one month no infected individuals were found. Split by parasite at Lloroza, for Bd persistence, Ia adults were the highest contributing host in two months, and Ia larvae in two. For Rv maintenance, Ia adults were the highest contributing host in three months, Ia larvae in one month, and Bs metamorphs in one.

The identity of the maintenance host categories were not solely driven by the abundance of each host (Tables 4 and 5). In the four site $\times$ parasite combinations, 3 of 7, 3 of 5, 4 of 7, and 3 of 5 of the months had maintenance hosts (highest $R_{0}$ contribution) that were also the most abundant host (Bd in Ercina, Bd in Lloroza, Rv in Ercina, and Rv in Lloroza, respectively).

As outlined above, the range of values of $R_{0}$ in the vast majority of site-month-pathogen combinations

TABLE 2. Mean, minimum, and maximum of $R_{0}$ values calculated for the host-category with the highest mean $R_{0}$ contribution at Lake Ercina, in the Picos de Europa National Park, Spain for the specified parasite.

\begin{tabular}{|c|c|c|c|c|c|c|c|c|}
\hline \multirow[b]{2}{*}{ Month } & \multicolumn{4}{|c|}{$\mathrm{Bd}$} & \multicolumn{4}{|c|}{$\mathrm{Rv}$} \\
\hline & Host-category & Mean & Range & $n$ & Host-category & Mean & Range & $n$ \\
\hline March & Ao larva & 1.41 & $1.40-1.41$ & 3 & Ao larva & 1.35 & $1.30-1.41$ & 3 \\
\hline April & Bs adult/juvenile & 1.02 & $1.02-1.02$ & 13 & Ao larva & 1.99 & $1.98-1.99$ & 5 \\
\hline May & Ao larva & 2.63 & $2.62-2.64$ & 20 & Ao larva & 1.82 & $1.73-1.92$ & 22 \\
\hline June & Ao metamorph & 2.86 & $2.62-3.13$ & 15 & Ao metamorph & 1.13 & $1.13-1.13$ & 15 \\
\hline July & Ao adult/juvenile & 1.48 & $1.33-1.66$ & 4 & Ia larva & 1.03 & $1.00-1.07$ & 17 \\
\hline August & Ao adult/juvenile & 2.06 & 2.02-2.10 & 18 & Ao adult/juvenile & 1.02 & $1.00-1.04$ & 18 \\
\hline September & Ao adult/juvenile & 2.38 & $2.38-2.38$ & 19 & Ao adult/juvenile & 1.01 & $1.01-1.01$ & 19 \\
\hline
\end{tabular}

Notes: Boldface text represents months for which the same host-category (Ao, Alytes obstetricans; Bs, Bufo spinosus; Ia, Ichthyosaura alpestris; Lh, Lissotriton helveticus; Rt, Rana temporaria) was the maintenance host-category for both parasites (Bd, Batrachochytrium dendrobatidis; Rv, Ranavirus). $R_{0}$, community reproductive number. 
TABle 3. Mean, minimum, and maximum of $R_{0}$ values calculated for the host-category with the highest mean $R_{0}$ contribution at Lake Lloroza, in the Picos de Europa National Park, Spain for the specified parasite.

\begin{tabular}{|c|c|c|c|c|c|c|c|c|}
\hline \multirow[b]{2}{*}{ Month } & \multicolumn{4}{|c|}{$\mathrm{Bd}$} & \multicolumn{4}{|c|}{$\mathrm{Rv}$} \\
\hline & Host-category & Mean & Range & $n$ & Host-category & Mean & Range & $n$ \\
\hline May & Ia adult/juvenile & 1.17 & $1.17-1.17$ & 40 & Ia adult/juvenile & 1.49 & $1.47-1.51$ & 40 \\
\hline June & Ia adult/juvenile & 0.97 & $0.93-1.02$ & 38 & Bs metamorph & 1.91 & $1.01-4.20$ & 21 \\
\hline July & Ia larva & 1.04 & $1.03-1.05$ & 5 & Ia adult/juvenile & 3.45 & $2.18-5.89$ & 57 \\
\hline August & no infected individuals detected & & & & Ia adult/juvenile & 1.57 & $1.22-2.07$ & 12 \\
\hline September & Ia larva & 1.25 & $1.25-1.25$ & 5 & Ia larva & 1.73 & $1.57-1.92$ & 5 \\
\hline
\end{tabular}

Notes: Boldface text represents months for which the same host-category with the highest mean $R_{0}$ contribution within the community at Lloroza for the specified parasite. Host and parasite abbreviations are as in Table 2.

was above the key threshold of $R_{0}>1$. Further, the range of values obtained when the level of inter vs. intra-host-category transmission was varied generally fairly tight around the mean value (Tables 2 and 3). The notable exceptions were June and July for $\mathrm{Rv}$ in Lloroza, where ranges of 1.01-4.20 and 2.18-5.89, respectively, were obtained. These two ranges also had the highest $R_{0}$ estimates of 4.20 and 5.89. Combined these results highlight that these site-pathogen-month combinations were most sensitive to changes in relative levels of inter- compared to intra-host-category transmission.

\section{Taxonomic patterns in maintenance hosts between sites over time}

When data on host-category within a species were tallied within a month, there was a significant species effect in which species contributed most to the maintenance of infection. Of the 23 site-species-parasite combinations, in 12 Ao contributed most to the maintenance of parasite infection, in 9 Ia was the top contributor, and in 2 Bs had the highest mean $R_{0}$ value (Fig. 3). Sign tests suggest that the number of times Ao and Ia were identified as the maintenance host significantly varied from what would be expected if all species had the same probability of contributing to infection maintenance within a community (probability of Ao being highest in 12 of 23 $<0.001$; probability of Ia being highest in 9 of 23 $<0.001)$.

Within each site there was also significant species-level signal in which host species was identified as being most important in parasite persistence. At Ercina, in 12 of 14 month-parasite combinations, Ao were identified as the maintenance host species in parasite maintenance. At Lloroza, Ia was the host species with the highest $R_{0}$ value in 8 of 9 parasite-month combinations. Sign tests suggest that both of these counts represent a significant deviation from a random distribution of maintenance host species (for 12 of 14 at Ercina $P<0.001$, and 8/9 at Lloroza $P<0.001)$.
Comparison of the relative contribution to community $\mathbf{R}_{O}$ obtained from the "central $\mathrm{R}_{0}$ estimate" and the "conservative $\mathrm{R}_{0}$ estimate"

Qualitatively the $R_{0}$ contribution results obtained using both prevalence estimates were very similar. Across all combinations of site-parasite-months $(n=23)$, only four differences existed in which hostcategory was inferred to be the maintenance host. These were for the top contributors to $\mathrm{Bd}$ persistence in Ercina in June and August, and for Rv at Lloroza in July and August (results in Appendix S1: Tables S1 and S2). Notably, in all of these cases, the top contributing host-category when we used the central prevalence estimate had a prevalence of infection calculated from a sample size of $n=1$. Using the more conservative prevalence estimate (lower $95 \%$ confidence interval) resulted in a much lower prevalence estimates for those life stages with small sample sizes, and those host categories therefore had much less important roles in parasite persistence (lower $R_{0}$ values). We therefore suggest that conservative $R_{0}$ estimates are preferable for making broad interpretation of results, as they are less affected by prevalence estimates based on small sample sizes.

When aggregated into species bins, the count of species acting as top contributors to parasite persistence were again very similar (Appendix S1: Tables S1 and S2); there was significant non-randomness in which species contributed to parasite maintenance at both sites and in both combined. The absolute values of $R_{0}$, however, were much higher when the central prevalence estimate was used in Eq. 1. Whether these absolute values or those in the main text of these analyses are more accurate is hard to ascertain, but the overall patterns in the data remain unchanged.

We focus on interpreting the outputs of the models focused on environmental transmission as the results obtained and patterns observed between these model outputs and those when estimating $R_{0}$ assuming direct transmission of infection are qualitatively the same (Appendix S1: Tables S3 and S4). 


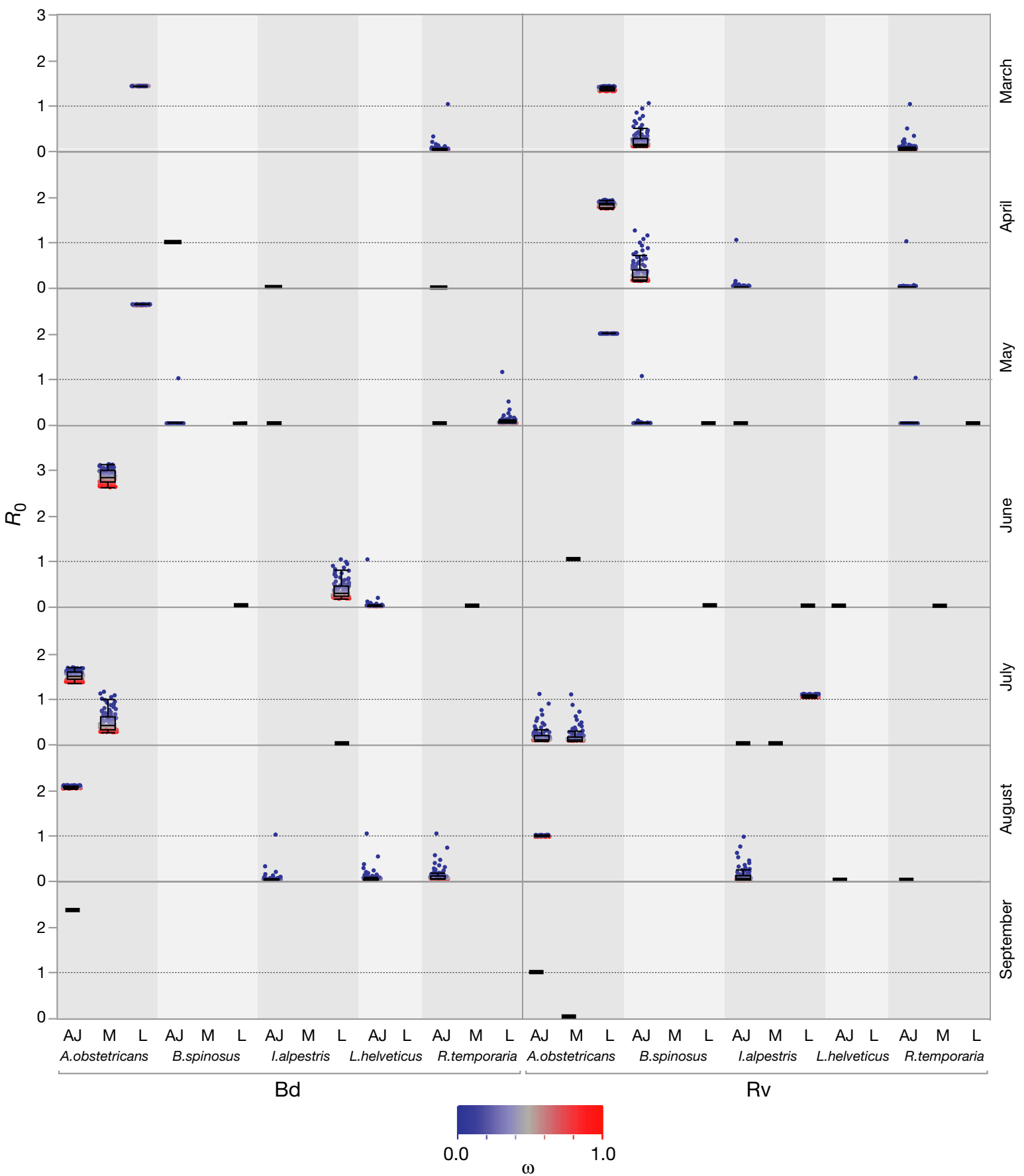

FIG. 1. Community reproductive number $\left(R_{0}\right)$ values obtained (left $y$-axis) for each host-category for each of two parasites, Ranavirus (Rv) and Batrachochytrium dendrobatidis (Bd; $x$-axis) sampled at Lake Ercina in the Picos de Europa National Park, Spain. Color of points correspond to the values of $\omega$, ranging from 0 (blue, at which no intra-host transmission occurred) to 1 (red, at which intra- and inter-host transmission were approximately equal). Taxa sampled are Alytes obstetricans, Bufo spinosus, Ichthyosaura alpestris, Lissotriton helveticus, and Rana temporaria. Abbreviations key: AJ, adult/juvenile; M, metamorphic individual; L, larva. Note that $y$-axis values vary across months.

\section{DisCUSSION}

Our analyses suggest that within a site and over the course of the year a single host species was responsible for the maintenance of our two focal parasites, Bd and Rv. The observed consistency did not extend between sites, which had different species associated with parasite persistence, both in those months in which infection 


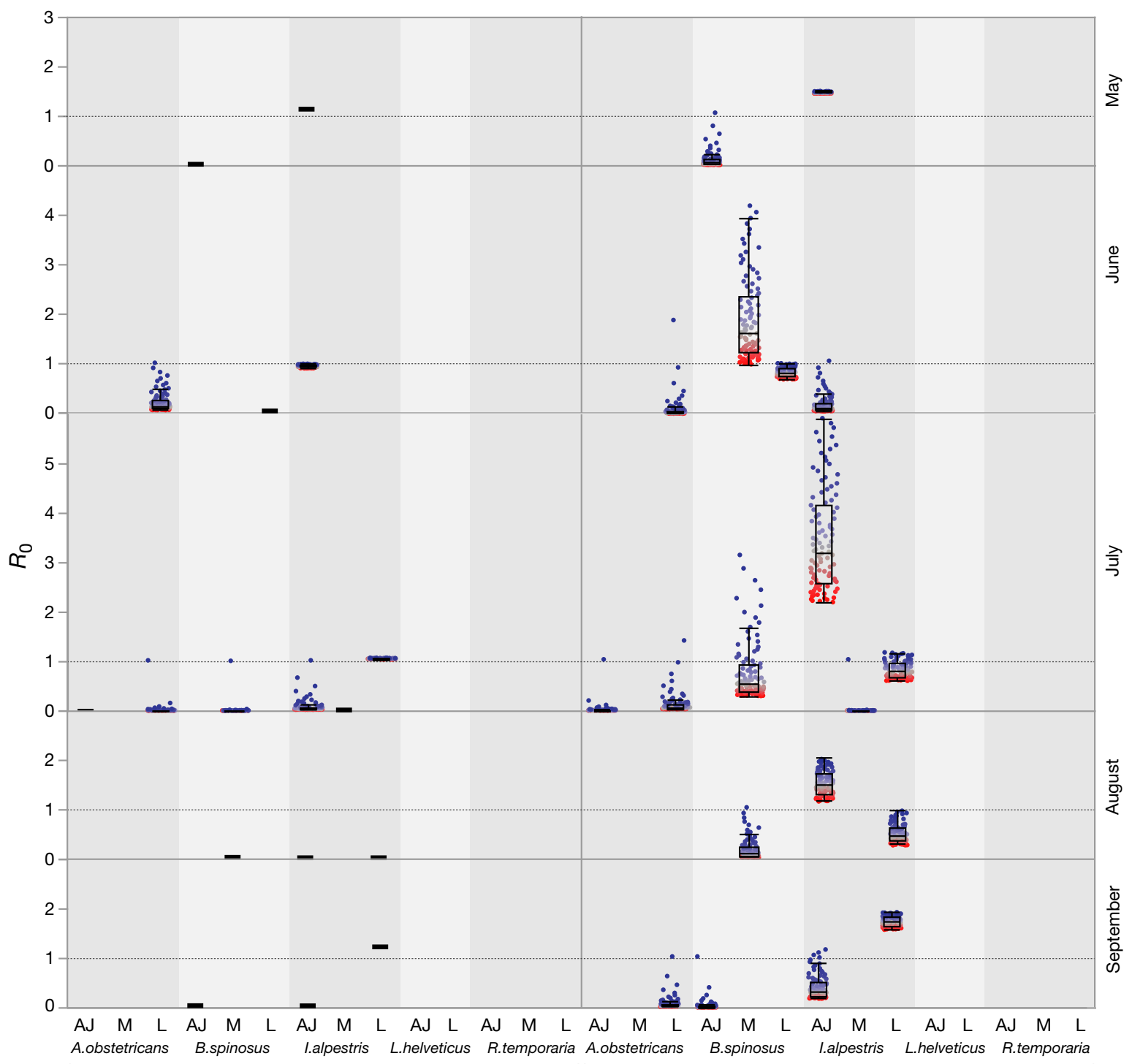

$\mathrm{Bd}$

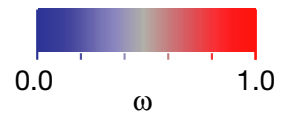

$\mathrm{Rv}$

FIG. 2. $R_{0}$ values obtained (left $y$-axis) for each host-category for each of two parasites, Rv and Bd ( $x$-axis), sampled at Lake Lloroza in the Picos de Europa National Park, Spain. Color of points correspond to the values of $\omega$, ranging from 0 (blue, at which no intra-host-category transmission occurred) to 1 (red, at which intra- and inter-host-category transmission were approximately equal). Taxa sampled are Alytes obstetricans, Bufo spinosus, Ichthyosaura alpestris, Lissotriton helveticus, and Rana temporaria. Abbreviations key: AJ, adult/juvenile; M, metamorphic individual; L, larva. Note that $y$-axis values vary across months.

levels are at equilibrium and, more generally, at sampling periods throughout the year. Further, our results are robust to changes in the amount of inter-specific transmission within the community with this parameter having negligible effect on which species is the maintenance host at a site. Here, we discuss these findings and their implications.

Despite their broad host ranges, both Bd and Rv largely rely on a single species for maintenance within the community. However, within a species the life stage contributing most to parasite persistence may vary throughout the year, likely due to the high seasonality of temperate amphibian life cycles. Knowing which host categories (i.e., species and life stages) contribute most to infection persistence is an important step in understanding and managing infections, particularly if a maintenance host is identified for multiple parasites. In some circumstances management efforts could "double their 
TABle 4. Summary data on the contingent variables used to calculate $R_{0}$ for both parasites in this study at Ercina (via Eq. 1).

\begin{tabular}{|c|c|c|c|c|c|c|c|c|c|c|}
\hline \multirow[b]{2}{*}{ Species } & \multirow[b]{2}{*}{ Life stage } & \multirow[b]{2}{*}{ Month } & \multicolumn{4}{|c|}{$\mathrm{Bd}$} & \multicolumn{4}{|c|}{$\mathrm{Rv}$} \\
\hline & & & Abundance & Mean $R_{0}$ & $n$ & Prevalence & Abundance & Mean $R_{0}$ & $n$ & Prevalence \\
\hline Ao & larva & 3 & 8 & $1.41 ; 97.69$ & 3 & 1 & 8 & $1.35 ; 87.47$ & 3 & 1 \\
\hline Bs & adult/juvenile & 3 & 10 & & 10 & 0 & 10 & & 10 & 0.2 \\
\hline Rt & adult/juvenile & 3 & 4 & & 4 & 0.25 & 4 & & 4 & 0.25 \\
\hline Ao & larva & 4 & 175 & & NA & NA & 175 & $1.99 ; 92.81$ & 5 & 1.00 \\
\hline Bs & adult/juvenile & 4 & 51 & $1.02 ; 1.08$ & 13 & 0.08 & 51 & & 13 & 0.46 \\
\hline Ia & adult/juvenile & 4 & 3 & & 1 & 0.00 & 3 & & 1 & 1.00 \\
\hline Rt & adult/juvenile & 4 & 7 & & 7 & 0.00 & 7 & & 7 & 0.14 \\
\hline Ao & larva & 5 & 4127 & $2.63 ; 6.62$ & 22 & 0.85 & 4127 & $1.82 ; 3.60$ & 22 & 0.73 \\
\hline Bs & adult/juvenile & 5 & 28 & & 14 & 0.07 & 28 & & 14 & 0.21 \\
\hline Bs & larva & 5 & 3 & & 3 & 0.00 & 3 & & 3 & 0.00 \\
\hline Ia & adult/juvenile & 5 & 3 & & 1 & 0.00 & 3 & & 1 & 0.00 \\
\hline Rt & adult/juvenile & 5 & 8 & & 4 & 0.00 & 8 & & 4 & 0.25 \\
\hline $\mathrm{Rt}$ & larva & 5 & 331 & & 19 & 0.32 & 331 & & 19 & 0.00 \\
\hline Ao & metamorph & 6 & 16 & 2.86 & 15 & 0.93 & 16 & $1.13 ; 1.49$ & 15 & 0.33 \\
\hline Bs & larva & 6 & 2 & & 2 & 0.00 & 2 & & 2 & 0.00 \\
\hline Ia & larva & 6 & 301 & 91.44 & 1 & 1.00 & 301 & & 1 & 0.00 \\
\hline $\mathrm{Lh}$ & adult/juvenile & 6 & 1 & & 1 & 1.00 & 1 & & 1 & 0.00 \\
\hline $\mathrm{Rt}$ & metamorph & 6 & 1 & & 1 & 0.00 & 1 & & 1 & 0.00 \\
\hline Ao & adult/juvenile & 7 & 5 & $1.48 ; 86.52$ & 4 & 1.00 & 5 & & 4 & 0.50 \\
\hline Ao & metamorph & 7 & 11 & & 11 & 0.36 & 11 & & 11 & 0.27 \\
\hline Ia & adult/juvenile & 7 & 1 & & NA & NA & 1 & & 0 & 0.00 \\
\hline $\mathrm{Ia}$ & larva & 7 & 112 & & 17 & 0 & 112 & $1.03 ; 1.24$ & 17 & 0.24 \\
\hline Ia & metamorph & 7 & 3 & & NA & NA & 3 & & 0 & 0.00 \\
\hline Ao & adult/juvenile & 8 & 12 & 2.06 & 12 & 0.06 & 12 & $1.01 ; 1.05$ & 12 & 0.17 \\
\hline Ia & adult/juvenile & 8 & 18 & & 18 & 1.00 & 18 & & 18 & 0.06 \\
\hline $\mathrm{Lh}$ & adult/juvenile & 8 & 2 & & 1 & 1.00 & 2 & & 1 & 0.00 \\
\hline Rt & adult/juvenile & 8 & 5 & 52.06 & 1 & 0.58 & 5 & & 1 & 0.00 \\
\hline Ao & adult/juvenile & 9 & 19 & $2.38 ; 2.38$ & 19 & 0.00 & 19 & $1.02 ; 1.05$ & 19 & 0.05 \\
\hline
\end{tabular}

Notes: Estimates of mean $R_{0}$ given are from calculations using lower prevalence estimate and central prevalence estimates, respectively (as described in Methods). Where these two estimates highlight different maintenance hosts, the $R_{0}$ calculated from the central prevalence estimate is given in italic type. Prevalence given in table is from lower prevalence estimate. Cells with boldface type represent combinations in which the most abundant host was identified as the maintenance host. Host and parasite abbreviations are as in Table 2. NA, no samples were taken at this point.

money"; by targeting a single maintenance species mitigation could potentially control two parasites, although the efficacy of this approach would depend on the species in question, the interactions between the parasites, and how the ecology of the maintenance host affects the management options being implemented.

Our results highlight how strongly the ecology of the host influences its ability to act as a maintenance host for parasite persistence, and how this may differ between ecological contexts (e.g., sites). At Lake Ercina the maintenance host was $A$. obstetricans (Ao), a species of conservation concern that has been heavily affected by $\mathrm{Bd}$ infection in the past two decades and has experienced numerous declines and even local extinction in parts of its range (Bosch et al. 2001). This species has a larval stage that, depending on conditions, can overwinter for multiple years. Overwintering anuran larvae have been implicated in the persistence of both Rv (Brunner et al. 2004) and Bd (Narayan et al. 2014, Fernández-Beaskoetxea et al. 2016), and have also been identified as a source of spillover to other hosts (Clare et al. 2016). Our analyses support these findings that in certain ecological conditions overwintering larvae can act as a maintenance host for infection within a community and therefore can make a suitable target for mitigation efforts (Woodhams et al. 2011, Bosch et al. 2015, Garner et al. 2016).

Parasite control, however, should be driven by the specifics of the focal site and our analyses underline how the ecology of the target maintenance hosts may vary greatly, with obvious implications of infection persistence and management. At Lloroza, the maintenance host was identified as Ichthyosaura alpestris (Ia), which contrasts strikingly with Ao. Rather than having longterm, solely aquatic life stages, Ia has been identified as being able to shift habitat on a short-term basis, with implications for its infection levels (Daversa et al. 2018). The species is also considered to be invasive in many parts of Europe, potentially acting as a carrier of $\mathrm{Bd}$ (Palomar et al. 2017). The adult life stage of this species can shift between aquatic and terrestrial habitats on a 
TABLE 5. Summary data on the contingent variables used to calculate $R_{0}$ for both parasites in this study at Lloroza (via Eq. 1).

\begin{tabular}{|c|c|c|c|c|c|c|c|c|c|c|}
\hline \multirow[b]{2}{*}{ Species } & \multirow[b]{2}{*}{ Life stage } & \multirow[b]{2}{*}{ Month } & \multicolumn{4}{|c|}{$\mathrm{Bd}$} & \multicolumn{4}{|c|}{$\mathrm{Rv}$} \\
\hline & & & Abundance & Mean $R_{0}$ & $n$ & Prevalence & Abundance & Mean $R_{0}$ & $n$ & Prevalence \\
\hline Ao & larva & 5 & 9 & & NA & NA & 9 & & 9 & 0.33 \\
\hline Bs & adult/juvenile & 5 & 73 & $1.17 ; 1.39$ & 40 & 0.29 & 73 & $1.49 ; 1.91$ & 40 & 0.50 \\
\hline $\mathrm{Rt}$ & adult/juvenile & 6 & 8 & 0.97 & 8 & 0 & 8 & & 8 & 0.88 \\
\hline Ao & larvae & 6 & 5252 & & 20 & 0.079 & 5252 & & 20 & 0.15 \\
\hline Bs & adult/juvenile & 6 & 40 & & NA & NA & 40 & $1.19 ; 4.53$ & 20 & 0.95 \\
\hline $\mathrm{Ia}$ & adult/juvenile & 6 & 169 & 1.01 & 38 & 0 & 169 & & 38 & 0.18 \\
\hline Rt & adult/juvenile & 7 & 16 & & 1 & 0.33 & 16 & 12.45 & 1 & 1.00 \\
\hline Ao & larvae & 7 & 13 & & 3 & 0.07 & 13 & & 3 & 1.00 \\
\hline Bs & adult/juvenile & 7 & 38 & & 15 & 0.05 & 38 & & 15 & 0.93 \\
\hline Bs & larvae & 7 & 136 & & 57 & 0.40 & 136 & 3.45 & 57 & 0.93 \\
\hline Ia & adult/juvenile & 7 & 1149 & $1.04 ; 1.65$ & 5 & 0.00 & 1149 & & 5 & 0.60 \\
\hline Rt & adult/juvenile & 7 & 2 & & 1 & 0.00 & 2 & & 1 & 1.00 \\
\hline Rt & larvae & 8 & 8 & & 3 & 0.00 & 8 & & 3 & 0.67 \\
\hline Ao & metamorph & 8 & 18 & & 12 & 0.00 & 18 & 1.57 & 12 & 0.83 \\
\hline Bs & larvae & 8 & 96 & & 1 & 0.00 & 96 & 94.65 & 1 & 1.00 \\
\hline Ia & larvae & 9 & 2 & & NA & NA & 2 & & 1 & 1.00 \\
\hline $\mathrm{Lh}$ & adult/juvenile & 9 & 1 & & 1 & 0.00 & 1 & & 1 & 1.00 \\
\hline $\mathrm{Rt}$ & metamorph & 9 & 8 & & 5 & 0.20 & 8 & & 5 & 0.60 \\
\hline Ao & adult/juvenile & 9 & 12 & $1.25 ; 1.25$ & 5 & 0.2 & 12 & $1.73 ; 71.32$ & 5 & 1.00 \\
\hline
\end{tabular}

Notes: Estimates of $R_{0}$ given are from calculations using lower prevalence estimate and central prevalence estimates respectively (as described in Methods). Where these highlight different maintenance hosts, the $R_{0}$ calculated from the central prevalence estimate is given in italics. Prevalence given in table is from lower prevalence estimate. Cells with boldface type represent combinations in which the most abundant host was identified as the maintenance host. Host and parasite abbreviations are as in Table 2.

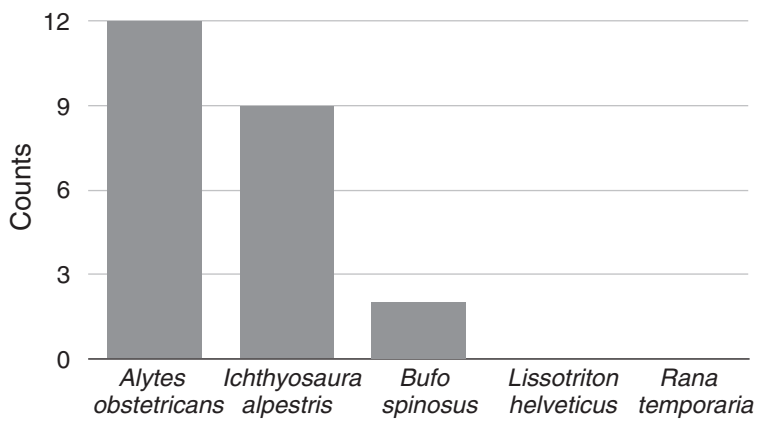

FIG. 3. Count of the number of times each species was the maintenance host (i.e., had the highest $R_{0}$ value) tallied over all site-parasite-month combinations.

day-to-day basis, which has the effect of "managing" their infection prevalence and intensity; by reducing contact with aquatic zoospores Ia may use habitat shifting as a means to lower infection levels, which, in turn, has been linked to a reduced role as a maintenance host within the community (Daversa et al. 2018). If habitat shifting, with increase terrestrial activity favors reduced infection levels, how would Ia play a key role in parasite persistence at Lloroza in this study? A potential answer to this question may lie in the impact of environmental drivers. Climatic conditions have been linked to the infection and disease dynamics in both Rv (Price et al. 2019) and Bd (Clare et al. 2016) and in the year of our data collection (2017) Southern Europe experienced an extreme weather event in the form of a heatwave. Although Lloroza did not dry out completely in this year, high air temperatures may have favored a more aquatic activity pattern in Ia to avoid the risk of desiccation. This reduced predisposition toward habitat-shifting would lead to increased duration of contact with aquatic zoospores and increased relative density of hosts in reduced water-levels. In combination these could increase infection levels and hence allow Ia to act as a maintenance host and allow $\mathrm{Bd}$ and $\mathrm{Rv}$ to persist. To test this prediction, multi-year sampling or experiments such as those outlined in Daversa et al. (2018) under different environmental conditions would be useful in disentangling the complex interactions between environment, host behavior, and the parasite infection patterns that we observe.

Our analyses suggest that single species acted as maintenance hosts at our focal sites, but in other situations, with more inter-host-category transmission, multiple hosts may play important roles in infection maintenance (Fenton and Pedersen 2005). Interestingly, Ao was historically present in relatively high numbers at Lloroza, but after years of mass-mortalities at the metamorphic stage the species is present only at very low density. Even with its generally high prevalence of infection at very low density the species and its age-classes seem to now play a relatively small role in parasite maintenance, although historically it could have been an important community host. Tracking the roles of different host in parasite maintenance as host abundance changed over 
time would be a fascinating route of future research. Doing so would allow us to answer outstanding questions such as whether the maintenance host identity changes over time; what community structures lead to parasite fade-out or persistence; and at what point in time is intervention best timed to maximize the chances of parasite control?

Given the broad host range of many parasites and the heterogeneity in which species contribute to parasite persistence, a key question to ask is whether the identity of the maintenance host varies when changes in transmission dynamics occur. Specifically, using sensitivity analyses, we quantified how shifts in inter- vs. intra-host life stage transmission rates altered the identity of maintenance hosts, and the extent to which they contributed to parasite persistence within a community. Our analyses suggest that the identity of the maintenance host (life stage or species) is robust of the relative levels of intercompared to intra-species or life-stage transmission. The values explored in our approach ranged from solely intra-life stage transmission $\left(\omega_{i j} \sim 0\right)$, to equal rates of inter and intra life stage transmission $\left(\omega_{i j} \sim 1\right)$. Regardless of the value of this parameter, the maintenance host-category remained the same; changing this parameter made no qualitative difference to the results obtained or their interpretation. Within some host-parasite systems there is relatively little inter-specific transmission despite the availability of many potential host species (Withenshaw et al. 2016). However, within the Bd-amphibian dynamics we do know that certain key hosts seem to amplify infection levels in other species (Fernández-Beaskoetxea et al. 2016). Our results here extend our knowledge in this area and suggest that even with high levels of interspecific transmission the community infection dynamics remain driven by a single host-category, the identity of which may vary between sites. While we know that many of the species present at our sites can be infected by and even suffer mortality as a result of both of these parasites (e.g., Bufo spinosus), the data suggest that at these sites, they are unlikely to act as maintenance hosts. The identity of the maintenance hosts here is perhaps not surprising, given their prominent role in parasite maintenance and spread in other locations and contexts.

While the identity of the maintenance hosts are robust to changes in the constituent parameter within the model (e.g., abundance, prevalence, and transmission rates), the absolute $R_{0}$ values obtained are sensitive to changes of constituent variables, and likely represent a conservative estimate of the actual number of secondary infections caused by a single host. Within these analyses we log-transformed our Bd infection intensity data (which we used as a measure of shedding rate) to account for extreme infection values obtained. Our justification for doing so is that while these values could represent real infection levels they lay orders of magnitude outside of the standards used in the assay, and so we chose to be conservative in our use of this data.
Similarly, we focused our analyses and interpretation on the conservative prevalence estimate, as outlined in the methods and results section. However, using the central prevalence estimate yielded qualitatively the same results, suggesting that the key points highlighted in this study remain unchanged regardless of the precise treatment of the raw infection data. Two very interesting paths for future research would be how the identity and relative contribution of maintenance hosts might vary with changes in host abundance levels, which may alter as a result of infection costs and disease-related mortalities. Our results (Tables 4 and 5) suggest that the maintenance host was not simply the most abundant hostcategory, although being a constituent variable, abundance does play an important role in infection dynamics. They key components of equation 1 are the prevalence of infection, the shedding rate of infected hosts, and the abundance. So, while the abundance (density) of infected hosts is important, the prevalence of infection within that category of hosts, and their rate of producing infectious particles are also important. In the context of classical epidemiological thinking, both contact rates (affected by abundance) and probability of infection per contact (affected by prevalence of infected hosts and their infection load), are key in quantifying the number of new infections, and this is reflected in the fact that our maintenance host wasn't always simply the category that was most abundant. The implications of this for management of infectious parasites in amphibians is that targeting the most abundant host-category will not always control infection spread and maintenance.

Further, understanding better how co-infection works within these sites and the relative levels of $R_{0}$ in coinfected vs. singly infected hosts could yield further insights into our understanding of the persistence of multiple parasites within a community.

The different ecologies of the maintenance hosts at our two sites highlight the variation in host-infection dynamics and therefore the different requirements of any mitigation efforts implemented. Such contrasts suggest that while understanding multi-host-parasite dynamics improves the information that we have in hand to tackle parasitic infections, they also show that onesize-fits-all management of such infections are challenging to develop. Although general rules can be useful (e.g., within a site, the maintenance species of multiple parasites may be the same), it is essential to obtain a deeper understand the biology of the system and the specifics of a site in order to better identify where those broad generalities are operationally useful. Doing so could ultimately result in the identification of key biotic and abiotic determinants that explain the maintenance of pathogen infection. Identifying these determinants should be the ultimate goal for developing general guidelines to control infection transmission in the broadest range of ecological contexts in which a given pathogen occurs. One of the biggest challenges in parasitemanagement and control is how to find the right balance 
between the efficiency of broad-scale, general rules of thumb, and having enough detailed context-dependent information to direct management options effectively. The trade-off between broad rules and system-specific details, when balanced correctly, can be used to provide useful coarse recommendations for fine-tuned management, taking into account the particulars of a given managed population or community.

The generality of this study is not universal, but it does provide a useful way to proceed in studies of multihost-multi-parasite dynamics. By identifying maintenance hosts, where they are consistent (within a site, between parasite and over time), and where they differ (between sites), we are able to better understand infection dynamics within this host-parasite system and better direct infection management. How likely it is that maintenance hosts will be the same for multiple parasites will likely vary according to host range, modes of transmission, and direct and indirect in-host interactions. However, by utilizing the commonly collected data (prevalence, host abundance, and infection shedding rate), we have illustrated how a recently developed modeling approach can highlight important trends in hostparasite dynamics, with a view to controlling infection persistence and the burden of infectious disease.

\section{ACKNOWLEDGMents}

This work has been funded by a MICINN grant (CGL201570070-R, PI: Jaime Bosch) and was possible thanks to the support from people working at Picos de Europa National Park, especially A. Mora and S. Marquínez. Author contributions: J. Bosch, J. Bielby, S. Price, and C. Monsalve-Carcaño conceived the ideas and designed field data collection; J. Bosch and C. Monsalve-Carcaño collected the data; J. Bielby, J. Bosch, and S. Price analysed the data; J. Bielby, J. Bosch, and S. Price led the writing of the manuscript. All authors contributed critically to the drafts and gave final approval for publication.

\section{Literature Cited}

Anderson, R. M., and R. M. May. 1992. Infectious diseases of humans: dynamics and control. Oxford University Press, Oxford, UK.

Balaž, V. et al 2014. Assessing risk and guidance on monitoring of Batrachochytrium dendrobatidis in Europe through identification of taxonomic selectivity of infection. Conservation Biology 28:213-223.

Barbour, A. 1996. Modeling the transmission of schistosomiasis: an introductory view. American Journal of Tropical Medicine and Hygiene 55:135-143.

Bates, K. A., F. C. Clare, S. O’Hanlon, J. Bosch, L. Brookes, K. Hopkins, E. J. McLaughlin, O. Daniel, T. W. Garner, and M. C. Fisher. 2018. Amphibian chytridiomycosis outbreak dynamics are linked with host skin bacterial community structure. Nature Communications 9:693.

Bosch, J., I. Martínez-Solano, and M. García-París. 2001. Evidence of a chytrid fungus infection involved in the decline of the common midwife toad (Alytes obstetricans) in protected areas of central Spain. Biological Conservation 97:331-337.

Bosch, J., E. Sanchez-Tomé, A. Fernández-Loras, J. A. Oliver, M. C. Fisher, and T. W. Garner. 2015. Successful elimination of a lethal wildlife infectious disease in nature. Biology Letters 11:20150874.

Boyle, D. G., D. B. Boyle, V. Olsen, J. A. T. Morgan, and A. D. Hyatt. 2004. Rapid quantitative detection of chytridiomycosis (Batrachochytrium dendrobatidis) in amphibian samples using real-time Taqman PCR assay. Diseases of Aquatic Organisms 60:141-148.

Brunner, J. L., D. M. Schock, E. W. Davidson, and J. P. Collins. 2004. Intraspecific reservoirs: complex life history and the persistence of a lethal ranavirus. Ecology 85:560-566.

Brunner, J. L., and C. M. Yarber. 2018. Evaluating the importance of environmental persistence for Ranavirus transmission and epidemiology. Pages 129-148 in Advances in virus research. Elsevier, Amsterdam, The Netherlands.

Clare, F. C., J. B. Halder, O. Daniel, J. Bielby, M. A. Semenov, T. Jombart, A. Loyau, D. S. Schmeller, A. A. Cunningham, and M. Rowcliffe. 2016. Climate forcing of an emerging pathogenic fungus across a montane multi-host community. Philosophical Transactions of the Royal Society B 371:20150454.

Daversa, D. R., A. Manica, J. Bosch, J. W. Jolles, and T. W. Garner. 2018. Routine habitat switching alters the likelihood and persistence of infection with a pathogenic parasite. Functional Ecology 32:1262-1270.

Duffus, A. L., T. B. Waltzek, A. C. Stöhr, M. C. Allender, M. Gotesman, R. J. Whittington, P. Hick, M. K. Hines, and R. E. Marschang. 2015. Distribution and host range of ranaviruses. Pages 9-57 in Ranaviruses. Springer, Cham, Switzerland.

Ezenwa, V. O., R. S. Etienne, G. Luikart, A. Beja-Pereira, and A. E. Jolles. 2010. Hidden consequences of living in a wormy world: nematode-induced immune suppression facilitates tuberculosis invasion in African buffalo. American Naturalist 176:613-624.

Fenton, A. 2008. Worms and germs: the population dynamic consequences of microparasite-macroparasite co-infection. Parasitology 135:1545-1560.

Fenton, A., and A. B. Pedersen. 2005. Community epidemiology framework for classifying disease threats. Emerging Infectious Diseases 11:1815.

Fenton, A., D. G. Streicker, O. L. Petchey, and A. B. Pedersen. 2015. Are all hosts created equal? Partitioning host species contributions to parasite persistence in multihost communities. American Naturalist 186:610-622.

Fernández-Beaskoetxea, S., J. Bosch, and J. Bielby. 2016. Infection and transmission heterogeneity of a multi-host pathogen (Batrachochytrium dendrobatidis) within an amphibian community. Diseases of Aquatic Organisms 118:11-20.

Funk, S., H. Nishiura, H. Heesterbeek, W. J. Edmunds, and F. Checchi. 2013. Identifying transmission cycles at the humananimal interface: the role of animal reservoirs in maintaining Gambiense human African trypanosomiasis. PLoS Computational Biology 9:e1002855.

Garmyn, A., P. Van Rooij, F. Pasmans, T. Hellebuyck, W. Van Den Broeck, F. Haesebrouck, and A. Martel. 2012. Waterfowl: potential environmental reservoirs of the chytrid fungus Batrachochytrium dendrobatidis. PLoS ONE 7:e35038.

Garner, T. W., B. R. Schmidt, A. Martel, F. Pasmans, E. Muths, A. A. Cunningham, C. Weldon, M. C. Fisher, and J. Bosch. 2016. Mitigating amphibian chytridiomycoses in nature. Philosophical Transactions of the Royal Society B 371:20160207.

Griffiths, E. C., A. B. Pedersen, A. Fenton, and O. L. Petchey. 2014. Analysis of a summary network of co-infection in humans reveals that parasites interact most via shared resources. Proceedings of the Royal Society B 281:20132286. 
Holt, R. D., A. P. Dobson, M. Begon, R. G. Bowers, and E. M. Schauber. 2003. Parasite establishment in host communities. Ecology Letters 6:837-842.

Hyatt, A. D. et al 2007. Diagnostic assays and sampling protocols for the detection of Batrachochytrium dendrobatidis. Diseases of Aquatic Organisms 73:175-192.

Jolles, A. E., V. O. Ezenwa, R. S. Etienne, W. C. Turner, and H. Olff. 2008. Interactions between macroparasites and microparasites drive infection patterns in free-ranging African buffalo. Ecology 89:2239-2250.

Knowles, S. C., A. Fenton, O. L. Petchey, T. R. Jones, R. Barber, and A. B. Pedersen. 2013. Stability of within-host-parasite communities in a wild mammal system. Proceedings of the Royal Society B 280:20130598.

Kolby, J. E., S. D. Ramirez, L. Berger, K. L. Richards-Hrdlicka, M. Jocque, and L. F. Skerratt. 2015. Terrestrial dispersal and potential environmental transmission of the amphibian chytrid fungus (Batrachochytrium dendrobatidis). PLoS ONE 10: $\mathrm{e} 0125386$.

Lembo, T., K. Hampson, D. T. Haydon, M. Craft, A. Dobson, J. Dushoff, E. Ernest, R. Hoare, M. Kaare, and T. Mlengeya. 2008. Exploring reservoir dynamics: a case study of rabies in the Serengeti ecosystem. Journal of Applied Ecology 45:1246-1257.

Leung, W. T., L. Thomas-Walters, T. W. Garner, F. Balloux, C. Durrant, and S. J. Price. 2017. A quantitative-PCR based method to estimate ranavirus viral load following normalisation by reference to an ultraconserved vertebrate target. Journal of Virological Methods 249:147-155.

Martel, A., F. Pasmans, M. C. Fisher, L. F. Grogan, L. F. Skerratt, and L. Berger 2018. Chytridiomycosis. Pages 309-335 in Emerging and epizootic fungal infections in animals. Springer International Publishing, Cham, Switzerland

Narayan, E. J., C. Graham, H. McCallum, and J.-M. Hero. 2014. Over-wintering tadpoles of Mixophyes fasciolatus act as reservoir host for Batrachochytrium dendrobatidis. PLoS ONE 9:e92499.

North, A. C., D. J. Hodgson, S. J. Price, and A. G. Griffiths. 2015. Anthropogenic and ecological drivers of amphibian disease (Ranavirosis). PLoS ONE 10:e0127037.

O'Hanlon, S. J., A. Rieux, R. A. Farrer, G. M. Rosa, B. Waldman, A. Bataille, T. A. Kosch, K. A. Murray, B. Brankovics, and M. Fumagalli. 2018. Recent Asian origin of chytrid fungi causing global amphibian declines. Science 360:621-627.

Palomar, G., J. Vörös, and J. Bosch. 2017. Tracking the introduction history of Ichthyosaura alpestris in a protected area of Central Spain. Conservation Genetics 18:867-876.

Pedersen, A. B., and A. Fenton. 2007. Emphasizing the ecology in parasite community ecology. Trends in Ecology \& Evolution 22:133-139.

Price, S. J., E. Ariel, A. Maclaine, G. M. Rosa, M. J. Gray, J. L. Brunner, and T. W. Garner. 2017. From fish to frogs and beyond: Impact and host range of emergent ranaviruses. Virology 511:272-279.

Price, S. J., T. W. Garner, R. A. Nichols, F. Balloux, C. Ayres, A.-M.-C. de Alba, and J. Bosch. 2014. Collapse of amphibian communities due to an introduced Ranavirus. Current Biology 24:2586-2591.

Price, S. J., W. T. Leung, C. J. Owen, R. Puschendorf, C. Sergeant, A. A. Cunningham, F. Balloux, T. W. Garner, and R. A. Nichols. 2019. Effects of historic and projected climate change on the range and impacts of an emerging wildlife disease. Global Change Biology 25:2648-2660.

R Core Team. 2019. R: a language and environment for statistical computing. R Foundation for Statistical Computing, Vienna, Austria. www.R-project.org

Rachowicz, L. J., and C. J. Briggs. 2007. Quantifying the disease transmission function: effects of density on Batrachochytrium dendrobatidis transmission in the mountain yellow-legged frog Rana muscosa. Journal of Animal Ecology 76:711-721.

Rosa, G. M., J. Sabino-Pinto, T. G. Laurentino, A. Martel, F. Pasmans, R. Rebelo, R. A. Griffiths, A. C. Stöhr, R. E. Marschang, and S. J. Price. 2017. Impact of asynchronous emergence of two lethal pathogens on amphibian assemblages. Scientific Reports 7:43260.

Rudge, J. W., J. P. Webster, D.-B. Lu, T.-P. Wang, G.-R. Fang, and M.-G. Basáñez. 2013. Identifying host species driving transmission of schistosomiasis japonica, a multihost parasite system, in China. Proceedings of the National Academy of Sciences USA 110:11457-11462.

Schmeller, D. S., M. Blooi, A. Martel, T. W. Garner, M. C. Fisher, F. Azemar, F. C. Clare, C. Leclerc, L. Jäger, and M. Guevara-Nieto. 2014. Microscopic aquatic predators strongly affect infection dynamics of a globally emerged pathogen. Current Biology 24:176-180.

Teacher, A., A. Cunningham, and T. Garner. 2010. Assessing the long-term impact of ranavirus infection in wild common frog populations. Animal Conservation 13:514-522.

Tobler, U., A. Borgula, and B. R. Schmidt. 2012. Populations of a susceptible amphibian species can grow despite the presence of a pathogenic chytrid fungus. PLoS ONE 7:e34667.

Tobler, U., and B. R. Schmidt. 2010. Within-and among-population variation in chytridiomycosis-induced mortality in the toad Alytes obstetricans. PLoS ONE 5:e10927.

Withenshaw, S. M., G. Devevey, A. B. Pedersen, and A. Fenton. 2016. Multihost Bartonella parasites display covert host specificity even when transmitted by generalist vectors. Journal of Animal Ecology 85:1442-1452.

Woodhams, D. C., J. Bosch, C. J. Briggs, S. Cashins, L. R. Davis, A. Lauer, E. Muths, R. Puschendorf, B. R. Schmidt, and B. Sheafor. 2011. Mitigating amphibian disease: strategies to maintain wild populations and control chytridiomycosis. Frontiers in Zoology 8:8. 